Errata

\title{
Facial features and axis of symmetry extracted using natural orientation information
}

\author{
L. Bowns', M. J. Morgan² \\ 1 Psychology Department, University of Nottingham, University Park, Nottingham NG7 2RD, UK \\ ${ }^{2}$ Laboratory for Neuroscience, Department of Pharmacology, University of Edinburgh Medical School, Appleton Tower 7, George Square, \\ Edinburgh EH8 9J2, UK
}

Biol. Cybern. 70, $137-144(1993)$

Acknowledgements. This study was supported by a grant from the SERC Image Interpretation Initiative.

We used image processing software written by $R$. Watt to process the MIRAGE part of our algorithm.

Due to an unfortunate error the above acknowledgements were not printed.

\section{A model of synchronization of motor acts to a stimulus sequence}

\section{Timing and error corrections \\ II. Stability analysis, error estimation and simulations}

\section{Jiří Mates}

Max Planck Institute for Psychological Research, Munich, Germany, Institute of Medical Psychology, Ludwig-Maximilians-University, Munich, Germany, and Institute of Physiology, Academy of Sciences of Czech Republic, Prague, Czech Republic

Biol. Cybern. 70, $463-473$ (1994)

Biol. Cybern. 70, 475 - 484 (1994)

Due to an unfortunate error one of the author's addresses was omitted. 